\section{Encoding the Universe}

\author{
Stephen Muggleton
}

The Universal Turing Machine: A Half-Century Survey. Edited by Rolf Herken. Oxford University Press: 1988. Pp. 674. £55, \$145

IN 1937 Alan Turing's description of a general purpose computing machine was published in the Proceedings of the London Mathematical Society. Computers based on Turing's ideas are a fact of modern life. But there is still scant awareness of the wide-ranging implications of the thesis behind his work, according to which a simple device with a limited internal memory and a movable, rewritable tape can compute every effectively calculable mathematical function.

At first sight Turing's thesis would appear to be a statement about mathematical functions. Indeed, this is the context in which Turing described his 'universal' machine. A year earlier, in 1936, Alonzo Church gave an account of a mathematical system, called $\lambda$ calculus, with which he showed that not all logically well-stated questions can be decided. According to Church, all effective computations could be carried out by manipulating formulae within the $\lambda$-calculus. Turing showed that his machine had the same computational power as Church's $\lambda$-calculus. The important difference between the two approaches
ON COMPITABLE NEMBERS, WITH AN APPLICATION TO THE ENTSCHEIDUN(SSPROBLEMI

$$
\text { By A. M. TrRixs. }
$$

Rerejed $2 x$ May, 1936. - Read 12 Nowember, 1936.

The "computable" numbers may be described briefly as the real numbers whose expressions as a decimal are calculable hy finite means. Although the subject of this paper is ostensibly the computable numbers. it is almost equally easy to define and investigate computable functions of an integral variable or a real or c predicates, and so forth. The funda however, the same in each case. and I hav for explicit treatment as involving the le shortly to give an account of the relati functions, and so forth to one another. of the theory of functions of a real var putable numbers. Aecording to $\mathrm{my}$ def if its decimal can be written down by a 1

In $\$ \$ 9.10$ I give some arguments with computable numbers include all num regarded as computable. In particular of numbers are computable. They inclu all algebraic numbers, the real parts of 1 the numbers $\pi$, e. etc. The computable 1 all definable numbers, and an example

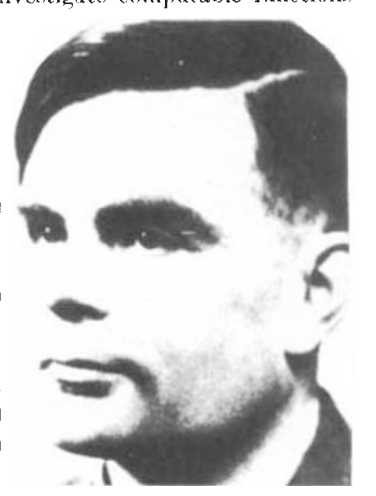

The second and main part of the book deals with applications of computation theory within a variety of scientific research areas. Among them is an account by Arbib of self-reproducing machines, an idea originated by von Neumann as a theoretical description of biological processes. These 'cellular automata' were designed not only to reproduce but to contain universal Turing machines for computing how to react to their environment.

A number of contributors discuss the problem of measuring physical complexity. Some physical structures and actions are more complex than others. For instance, imagine giving an adequate description of the structures of both a pure crystal, such as diamond, and a human body, when each contains an equal number of particles. Clearly, the repeated pattern of diamond allows a far simpler description. Kolmogorov, one of the founders of modern statistics, was the first to suggest that the absolute information content of an encoded description could be defined as being equal to the minimal length of a universal Turing machine program which exactly reproduces the given description. Among the researchers who have further developed this idea, two of the contributors, Chaitin and Bennett, have brought out the close relationship between minimal Turing machine descriptions on the one hand and statistical and physical notions on entropy on the other.

Turing himself was deeply interested in the use of digital is that whereas Church's $\lambda$-calculus it also provides an up-to-date source for represents a universal mathematical notation for calculation, Turing's universal machines form the vital link with physical computability.

Turing's thesis can be viewed as a claim not only about the limitations of mathematics but also about the limitations of physical process. If there were a physical process which could be used to compute a function which a universal Turing machine could not compute, the thesis would be false. If the thesis is true then there is no such process, and universal Turing machines are sufficient for modelling all of physics. To answer quibbles about the task of simulating, say, quantum mechanical components of such a process, David Deutsch (Proceedings of the Royal Society, London A 400,97-117; 1985) has provided an appropriate quantum-based 'fix' to universal Turing machines. Indeed our whole observable universe could be regarded as one huge, loosely coupled computational network. Furthermore, if we accept the physical basis of life processes, we are forced to conclude that computers could, in principle, simulate those interested in research on computation-theoretic models applicable to physics and biology. Among the authors of the survey are Stephen Kleene, Church's student and co-developer of $\lambda$ calculus, and Donald Michie, who worked with Turing in the Bletchley Park establishment for decoding messages during the Second World War.

The book is divided into two parts. In the first section Hodges, Kleene, Gandy, Feferman and Davis provide descriptions of the historical and theoretical background to Turing's work. There is some overlap with previous historical accounts, such as Hodges's biography of Turing, and overlap between the contributions themselves - thus we are repeatedly asked to pay heed to Leibniz's injunction, "Let us calculate". Nonetheless, a fair amount of material is new. For instance Davis's account of the development of mathematical logic and its relationship to modern computing should be stimulating for those interested in the history of computing, as Davis himself has played an important role within that history. computers for simulating human intelligence. However, not all authors in this volume believe the goals of machine intelligence to be entirely achievable. Notably, Roger Penrose uses Gödel's incompleteness theorem to argue that there may exist some as yet unknown physical laws which allow human beings to exhibit mental abilities which cannot be simulated by machines. Turing gave a simpler explanation. He suggested the possibility of a computer programmed to learn and permitted to make mistakes. In a lecture delivered in 1947 he stated that there "are several theorems which say almost exactly that ... if a machine is expected to be infallible, it cannot also be intelligent.... But these theorems say nothing about how much intelligence may be displayed if a machine makes no pretence at infallibility". These ideas form the basis of the rapidly evolving science of machine learning - according to Michie's article, the missing link in the Japanese Fifth Generation computer project.

Stephen Muggleton is a Research Fellow at the Turing Institute, George House, 36 North Hanover Street, Glasgow G1 2 AD, UK. 